\title{
Effect of myofascial release on electrophysiological and clinical measures of pregnant women with carpal tunnel syndrome
}

\author{
DOI: https://doi.org/10.5114/pq.2019.83057
}

\author{
Reham E. Hamoda', Doaa A. Osman', Hamada Ahmed Hamada², Rafik Radwan², Amel M. Yousef', \\ Gehan A. Abdel Samea', Hassan 0. Gharib ${ }^{3}$ \\ ${ }^{1}$ Department of Physical Therapy for Woman's Health, Faculty of Physical Therapy, Cairo University, Giza, Egypt \\ 2 Department of Biomechanics, Faculty of Physical Therapy, Cairo University, Giza, Egypt \\ ${ }^{3}$ Department of Obstetrics and Gynecology, Faculty of Medicine, Cairo University, Giza, Egypt
}

\section{Abstract}

Introduction. To explore the effect of myofascial release on electrophysiological and clinical measures of pregnant women with carpal tunnel syndrome (CTS).

Methods. Randomized controlled trial. Overall, 30 pregnant women with CTS, aged 25-35 years, were randomized into 2 equal groups. The control group only wore a neutral wrist splint during sleeping for 4 weeks, while the study group received a myofascial release in addition to wearing a neutral wrist splint during sleeping for 4 weeks. All pregnant women in both groups were evaluated pre- and post-treatment with median nerve distal motor latency and Boston Carpal Tunnel Questionnaire to assess pain, numbness, and tingling sensation severity, as well as hand function.

Results. Comparing both groups post-treatment revealed that there was a statistically highly significant decrease in median nerve distal motor latency, pain intensity, numbness, and tingling sensation severity, as well as in weakness and difficulty in grasping severity $(p<0.001)$ in favour of the study group.

Conclusions. Myofascial release is an effective method for improving electrophysiological and clinical measures of pregnant women with CTS.

Key words: myofascial release, carpal tunnel syndrome, pregnancy, median nerve distal motor latency, Boston Carpal Tunnel Questionnaire

\section{Introduction}

Carpal tunnel syndrome (CTS) is a common complication of pregnancy. The prevalence of CTS among pregnant women is relatively high; $19 \%$ of the pregnant women had CTS, with bilateral disease in $47.5 \%$ of them and severe CTS in $26.3 \%$. The reported prevalence in the first, second, and third trimesters were $11 \%, 26 \%$, and $63 \%$, respectively [1].

Water retention is the main cause of CTS during pregnancy. The symptoms commonly resolve after delivery. However, approximately $50 \%$ of the affected women may still experience mild symptoms after 3 years [2].

CTS can hinder the mother's ability to perform many daily tasks, including shopping, using cutlery, or even holding a phone up to her ear. In addition, the pain can radiate to the arm, shoulder, and neck, making hand and arm motions more uncomfortable. Moreover, it can contribute to sleep difficulties as many women report that their pain often worsens at night. Furthermore, it may affect the mother's ability to carry her infant if symptoms last after childbirth [3].

Pregnant women sometimes cannot receive any medications, such as non-steroidal anti-inflammatory drugs, which are not safe during pregnancy and therefore should not be used to treat CTS in that period [4].

The contents of the carpal tunnel should be assessed with a modified range of motion procedures reported by Sucher [5], which were designed for the evaluation of so- matic dysfunction. Patients are examined in the seated position with the wrist flexed to right angle. Restrictions of motion are graded from 0 to 5 , with 0 denoting no restriction, 1 - mild restriction, 2 - moderate restriction, 3 - moderate to marked restriction, 4 - marked restriction, and 5 extremely marked restriction.

Myofascial release is a method of diagnosis and treatment, first designated by Andrew Taylor Still, which involves persistently palpatory feedback to attain release of myofascial tissues. There are two techniques of application, direct and indirect. A full understanding of upper limb anatomy plays a crucial role in clarifying the aetiology of CTS. Osteopathic structural examination helps to identify somatic dysfunctions, defined as altered or impaired body structures that include skeletal, arthrodial, myofascial, vascular, lymphatic, and neural components [6].

This study presents a trial to conduct a new physiotherapy intervention (myofascial release) that may lead to improvement of electrophysiological and clinical measures in pregnant women with CTS.

\section{Subjects and methods}

\section{Design}

The study was designed as a prospective, randomized, controlled trial. It was conducted between May 2013 and

Correspondence address: Hamada Ahmed, Department of Biomechanics, Faculty of Physical Therapy, Cairo University,

7 Ahmed Elziat Street, Bean Elsariat, El Dokki, Giza, Cairo, Egypt, e-mail: Hamada.Ahmed@pt.cu.edu.eg

Received: 11.01 .2019

Accepted: 30.01 .2019

Citation: Hamoda RE, Osman DA, Hamada HA, Radwan R, Yousef AM, Abdel Samea GA, Gharib HO. Effect of myofascial release on electrophysiological and clinical measures of pregnant women with carpal tunnel syndrome. Physiother Quart. 2019;27(1):18-24; doi: https://doi. org/10.5114/pq.2019.83057. 
April 2014 and followed the Consolidated Standards of Reporting Trials guidelines for reporting randomized controlled trials.

\section{Participants}

A sample of 30 pregnant women were recruited from the Obstetric Outpatient Clinic, El-Sahel Teaching Hospital, Cairo, Egypt. The inclusion criteria were early third trimester pregnancy, idiopathic CTS complaints (pain, numbness, and tingling of the hand) confirmed by electrophysiological examination, i.e. delayed median nerve distal motor latency $(\mathrm{DML})>3.9 \mathrm{~ms}$, as well as positive Phalen's test. Pregnant women having bilateral and unilateral CTS affection participated but the dominant hand data only were enrolled in the study. The subjects' age ranged from 25 to 35 years, their body mass index (BMI) did not exceed $34 \mathrm{~kg} / \mathrm{m}^{2}$, and their gravidity number ranged from 1 to 3 . The participants were excluded if they had other predisposing causes for CTS and/or neuromuscular diseases that might affect median nerve transmission, such as diabetes mellitus, pre-eclampsia, rheumatoid arthritis, previous CTS symptoms, acute hand trauma, cervical spondylosis, previous surgeries in the forearm involving the median nerve, peripheral neuropathy, or pronator teres syndrome. All pregnant women did not receive any physical or medical therapy for treating CTS complains and had no serious medical problems such as a pacemaker or heart diseases that might interfere with electrophysiological testing.

\section{Randomization}

Each participant was informed about the nature, purpose, and benefits of the study, the right to refuse or withdraw at any time, and the confidentiality of any obtained data. The pregnant women were randomly assigned into 2 equal groups (control group and study group) with the use of a computerbased randomization program. No dropping out of subjects from the study was reported after randomization.

\section{Interventions}

The control group was composed of 15 pregnant women who only wore a neutral wrist splint during sleeping for 4 weeks. The study group involved 15 pregnant women who received a myofascial release in addition to wearing a neutral wrist splint during sleeping for 4 weeks.

\section{Neutral wrist splint}

A neutral wrist splint was worn by all pregnant women in both groups at night all through the study time (4 weeks). The neutral wrist splint was used to keep the wrist in a straight (neutral) position and to prevent extreme wrist motion (flexion and extension) while sleeping.

\section{Myofascial release technique}

In each pregnant woman in the study group, myofascial release technique was applied, 3 sessions per week for 4 weeks. The position for treatment was long sitting with the back fully supported, the dominant palm and forearm exposed and rested comfortably. The myofascial release technique was divided into two parts:

- First part: myofascial wrist retinaculum (transverse carpal ligament) release. The physical therapist placed her fourth and fifth fingers of both hands between the pregnant woman's fourth and fifth fingers and first and second fingers of the palmar surface and extension of the pregnant woman's wrist was introduced. The physical therapist's thumbs overlay the lateral and medial attachments of the wrist flexor retinaculum and transverse distraction to the retinaculum was applied until relaxation of the soft tissue or release of the restriction was achieved (for about 30 seconds); then, relaxation time was about 10 seconds. A total of 15 repetitions were performed in each session, 3 sessions per week for 4 weeks.

- Second part: interosseous membrane and forearm muscles myofascial release (bilateral thumb pressure technique). Gentle force was applied ventrally on the forearm through 2 hands grasping the pregnant woman's distal forearm with the thumbs contacting the anterior aspect of the forearm muscles and interosseous membrane while the index fingers grasping the posterior aspect of the forearm to engage the soft tissues cephalad until a barrier of tissue motion was reached and held for about 30 seconds; then, relaxation time was about 10 seconds. A total of 15 repetitions were performed in each session, 3 sessions per week for 4 weeks.

\section{Outcome measures}

\section{Median nerve DML}

It was assessed in all the pregnant women in both groups before participation in the study to confirm their diagnosis of CTS and also after the end of the treatment.

Each subject in both groups was asked to evacuate her bladder to be more relaxed and then she was informed about the aim and steps of the test. Each woman was seated on a wooden chair and her back was supported. The dominant forearm was in supinated position, rested on the examining table, and the elbow joint was held slightly flexed. The pregnant woman's body temperature was measured with an oral mercury thermometer and if it was normal, the test procedure was carried out. Also, the room temperature was adjusted at $22^{\circ} \mathrm{C}$ and controlled by an air conditioner as median nerve DML could be affected by the participant temperature as well as the room temperature.

Before electrode placement, the areas used for recording, stimulating, or ground points were cleaned with alcohol to decrease skin resistance. Further reduction of impedance was obtained by applying a conducting gel on the surface of the recording electrodes.

Then, the electromyography apparatus was calibrated and the parameters were adjusted as follows: time base at $5.0 \mathrm{~ms} /$ division, sensitivity at $4000.0 \mathrm{~V} /$ division, and intensity depending on the site of stimulation (about $7 \mathrm{~mA}$ for wrist stimulation and $12 \mathrm{~mA}$ for elbow stimulation) [7].

After the preparation, the active recording electrode was placed on the motor point of the abductor pollicis brevis muscle and the reference recording electrode on the tip of the thumb. They were fixed to the hand by adhesive plaster straps. The bipolar stimulating electrode was placed above the wrist joint, between the tendons of the palmaris longus and the flexor carpi radialis muscles, on the course of the median nerve, with the negative pole distal toward the active recording electrode, and the positive pole proximal to stimulate the median nerve. The ground electrode was placed on the distal wrist crease midway between the stimulating and recording electrodes; $7 \mathrm{~mA}$ were used for wrist stimulation.

Then, the bipolar stimulating electrode was placed in the cubital fossa, just medial to the biceps tendon, with the negative pole distal toward the active recording electrode, and 
the positive pole proximal. The recording electrodes were applied at the same locations as in recording at the wrist level; $12 \mathrm{~mA}$ were used for elbow stimulation.

\section{Boston Carpal Tunnel Questionnaire}

It was self-applied and evaluated the severity of symptoms and the functional status of patients with CTS. It had a comprehensive framework, good validity, reliability, and responsiveness in the hands of the researchers [8]. The questionnaire comprised two scales, a symptom severity scale (SSS) and a functional status scale (FSS). The SSS evaluated symptoms with regard to severity, frequency, and time. The FSS indicated how the syndrome affected daily life. SSS involved 11 questions, addressing: pain intensity during day time and night time, time of pain during the day, numbness, weakness, tingling sensation at night, frequency of night tingling sensation, and skill. Each question offered 5 answers, from 0 points (no symptoms) to 4 points (most severe symptoms). Questions concerning FSS referred to daily activities that were performed by most individuals and were commonly affected by CTS. The patients rated their ability to perform each activity on a scale that ranged from 1 point (no difficulty with the activity) to 5 points (cannot perform the activity at all). The overall score for the FSS was the mean of the ratings on the daily activities. This questionnaire was performed before and after treatment for both groups.

\section{Statistical analysis}

The sample size of 30 patients was calculated to yield an $80 \%$ power and $\alpha=0.05$. Results are expressed as mean \pm standard deviation or median (minimum-maximum). The mean median difference was established as follows:

$$
\text { difference }=\text { pre-treatment }- \text { post-treatment }
$$

The comparison between mean values of variables in the 2 groups (control and study) was performed with unpaired $t$-test, while pairwise comparison (pre-treatment vs. post- treatment) within the same group involved paired $t$-test. The comparison between median values in the 2 groups (control and study) was performed with Mann-Whitney $U$ test, while pairwise comparison (pre-treatment vs. post-treatment) within the same group involved the Wilcoxon signed rank test. The Statistical Package for the Social Sciences (SPSS) computer program (version 19 for Windows) was applied for data analysis. The value of $p \leq 0.05$ was considered significant and $p<0.01$ was considered highly significant.

\section{Ethical approval}

The research related to human use has been complied with all the relevant national regulations and institutional policies, has followed the tenets of the Declaration of Helsinki, has been approved by the Research Ethics Committee of the Faculty of Physical Therapy, Cairo University, and has been registered at ClinicalTrial.gov (identifier: NCT03802448).

\section{Informed consent}

Informed consent has been obtained from all individuals included in this study.

\section{Results}

A total of 30 pregnant women were randomized for the study intervention. The control group consisted of 15 pregnant women who only wore a neutral wrist splint during sleeping for 4 weeks. The study group consisted of 15 pregnant women who received a myofascial release in addition to wearing a neutral wrist splint during sleeping for 4 weeks. All participants completed the trial, as shown in Figure 1. The groups were similar at baseline $(p>0.05)$ with regard to age, weight, height, BMI, and the outcome measures (Tables 1-5).

The median nerve DML showed a statistically non-significant difference $(p>0.5)$ within the control group, while it presented a statistically highly significant decrease $(p<$ 0.001 ) within the study group. The post-treatment comparison of both groups revealed a statistically non-significant difference $(p>0.5)$. The comparison of the mean difference between the pre-treatment and post-treatment mean val-

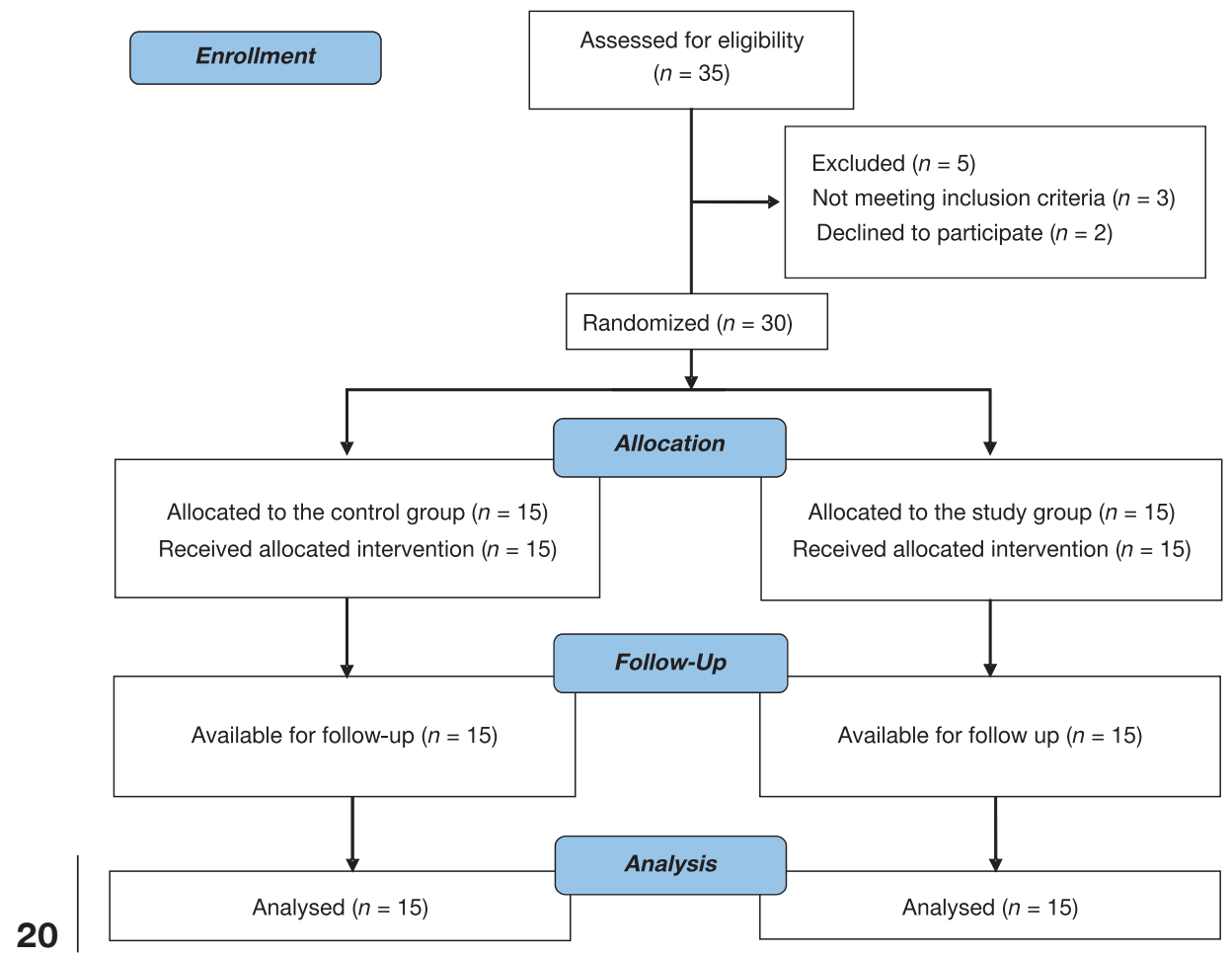

Figure 1. The flow chart of the randomized trial in accordance with the CONSORT Statement 
Table 1. Demographic data of the pregnant women in both groups

\begin{tabular}{|c|c|c|c|c|c|c|c|}
\hline \multirow{2}{*}{ Variables } & \multirow{2}{*}{ Groups } & \multicolumn{2}{|c|}{ Range } & \multirow{2}{*}{ Mean } & \multirow{2}{*}{$S D$} & \multirow{2}{*}{$t$ value } & \multirow{2}{*}{$p$ value } \\
\hline & & minimum & maximum & & & & \\
\hline \multirow{2}{*}{ Age (years) } & control & 25 & 35 & 29.53 & \pm 2.85 & \multirow{2}{*}{-0.565} & \multirow{2}{*}{$>0.05$} \\
\hline & study & 25 & 35 & 30.20 & \pm 3.57 & & \\
\hline \multirow{2}{*}{ Weight (kg) } & control & 73 & 91 & 82.67 & \pm 4.62 & \multirow{2}{*}{0.194} & \multirow{2}{*}{$>0.05$} \\
\hline & study & 70 & 89 & 82.33 & \pm 4.79 & & \\
\hline \multirow{2}{*}{ Height (cm) } & control & 151 & 164 & 161.0 & \pm 3.61 & \multirow{2}{*}{1.511} & \multirow{2}{*}{$>0.05$} \\
\hline & study & 154 & 165 & 158.93 & \pm 3.88 & & \\
\hline \multirow{2}{*}{$\mathrm{BMI}\left(\mathrm{kg} / \mathrm{m}^{2}\right)$} & control & 29.74 & 33.83 & 31.88 & \pm 1.20 & \multirow{2}{*}{-1.852} & \multirow{2}{*}{$>0.05$} \\
\hline & study & 30.48 & 33.98 & 32.64 & \pm 1.04 & & \\
\hline
\end{tabular}

$\mathrm{BMI}$ - body mass index

Table 2. Median nerve DML for both groups

\begin{tabular}{|c|c|c|c|c|}
\hline \multirow{3}{*}{ Variables } & \multicolumn{4}{|c|}{ Median nerve DML (ms) } \\
\hline & \multicolumn{2}{|c|}{ control group $(n=15)$} & \multicolumn{2}{|c|}{ study group $(n=15)$} \\
\hline & pre-treatment & post-treatment & pre-treatment & post-treatment \\
\hline Mean & 4.72 & 4.69 & 4.67 & 4.32 \\
\hline SD & \pm 0.93 & \pm 0.94 & \pm 0.80 & \pm 0.70 \\
\hline Mean difference & \multicolumn{2}{|c|}{0.03} & \multicolumn{2}{|c|}{0.35} \\
\hline$\%$ of improvement & \multicolumn{2}{|c|}{$0.64 \%$} & \multicolumn{2}{|c|}{$7.5 \%$} \\
\hline$t$ value & \multicolumn{2}{|c|}{1.000} & \multicolumn{2}{|c|}{5.426} \\
\hline$p$ value & \multicolumn{2}{|c|}{$>0.05$} & \multicolumn{2}{|c|}{$<0.001$} \\
\hline
\end{tabular}

$\mathrm{DML}$ - distal motor latency

Table 3. CTS pain intensity measure by BCTQ in both groups

\begin{tabular}{|c|c|c|c|c|c|c|c|c|}
\hline \multirow{3}{*}{ Intensity of CTS pain by BCTQ } & \multicolumn{4}{|c|}{ Control group $(n=15)$} & \multicolumn{4}{|c|}{ Study group $(n=15)$} \\
\hline & \multicolumn{2}{|c|}{ pre-treatment } & \multicolumn{2}{|c|}{ post-treatment } & \multicolumn{2}{|c|}{ pre-treatment } & \multicolumn{2}{|c|}{ post-treatment } \\
\hline & No. & $\%$ & No. & $\%$ & No. & $\%$ & No. & $\%$ \\
\hline No pain & 0 & & 0 & & 0 & & 11 & 73.33 \\
\hline Little pain & 1 & 6.67 & 2 & 13.33 & 3 & 20 & 4 & 26.67 \\
\hline Moderate pain & 8 & 53.33 & 11 & 73.33 & 8 & 53.33 & 0 & \\
\hline Intense pain & 6 & 40 & 2 & 13.33 & 4 & 26.67 & 0 & \\
\hline Sever pain & 0 & & 0 & & 0 & & 0 & \\
\hline Range & \multicolumn{2}{|c|}{$1-3$} & \multicolumn{2}{|c|}{$1-3$} & \multicolumn{2}{|c|}{$1-3$} & \multicolumn{2}{|c|}{$0-1$} \\
\hline Median & \multicolumn{2}{|c|}{2} & \multicolumn{2}{|c|}{2} & \multicolumn{2}{|c|}{2} & \multicolumn{2}{|c|}{0} \\
\hline$z$ value & \multicolumn{4}{|c|}{-1.890} & \multicolumn{4}{|c|}{-3.482} \\
\hline$p$ value & \multicolumn{4}{|c|}{$>0.05$} & \multicolumn{4}{|c|}{$<0.001$} \\
\hline
\end{tabular}

CTS - carpal tunnel syndrome, BCTQ - Boston Carpal Tunnel Questionnaire

ues of the control group vs. the study group proved a statistically highly significant decrease in median nerve DML $(p<0.001)$ in favour of the study group (Table 2$)$.

The pain intensity showed a statistically non-significant difference $(p>0.5)$ within the control group and a statistically highly significant decrease $(p<0.001)$ within the study group. The post-treatment comparison of both groups revealed a statistically highly significant decrease in pain intensity $(p<0.001)$ in favour of the study group (Table 3 ).
The numbness and tingling sensation severity showed a statistically highly significant decrease $(p<0.001)$ within both groups. Also, the post-treatment comparison of both groups revealed a statistically highly significant decrease in the numbness and tingling sensation severity $(p<0.001)$ in favour of the study group (Table 4).

The weakness and difficulty in grasping severity showed a statistically non-significant difference $(p>0.5)$ within the control group and a statistically highly significant decrease 
Table 4. CTS numbness and tingling sensation severity measure by BCTQ in both groups

\begin{tabular}{|c|c|c|c|c|c|c|c|c|}
\hline \multirow{3}{*}{$\begin{array}{l}\text { Numbness and tingling sensation severity } \\
\text { by BCTQ }\end{array}$} & \multicolumn{4}{|c|}{ Control group $(n=15)$} & \multicolumn{4}{|c|}{ Study group $(n=15)$} \\
\hline & \multicolumn{2}{|c|}{ pre-treatment } & \multicolumn{2}{|c|}{ post-treatment } & \multicolumn{2}{|c|}{ pre-treatment } & \multicolumn{2}{|c|}{ post-treatment } \\
\hline & No. & $\%$ & No. & $\%$ & No. & $\%$ & No. & $\%$ \\
\hline No numbness or tingling sensation & 0 & & 0 & & 0 & & 9 & 60 \\
\hline Little numbness and tingling sensation & 0 & & 7 & 46.67 & 0 & & 6 & 40 \\
\hline Moderate numbness and tingling sensation & 7 & 46.67 & 8 & 53.33 & 7 & 46.67 & 0 & \\
\hline Intense numbness and tingling sensation & 5 & 33.33 & 0 & & 6 & 40 & 0 & \\
\hline Severe numbness and tingling sensation & 3 & 20 & 0 & & 2 & 13.33 & 0 & \\
\hline Range & \multicolumn{2}{|c|}{$2-4$} & \multicolumn{2}{|c|}{$1-2$} & \multicolumn{2}{|c|}{$2-4$} & \multicolumn{2}{|c|}{$0-1$} \\
\hline Median & \multicolumn{2}{|c|}{3} & \multicolumn{2}{|c|}{2} & \multicolumn{2}{|c|}{3} & \multicolumn{2}{|c|}{0} \\
\hline$z$ value & \multicolumn{4}{|c|}{-3.286} & \multicolumn{4}{|c|}{-3.578} \\
\hline$p$ value & \multicolumn{4}{|c|}{$<0.001$} & \multicolumn{4}{|c|}{$<0.001$} \\
\hline
\end{tabular}

CTS - carpal tunnel syndrome, BCTQ - Boston Carpal Tunnel Questionnaire

Table 5. CTS weakness and difficulty in grasping severity measure by BCTQ in both groups

\begin{tabular}{|c|c|c|c|c|c|c|c|c|}
\hline \multirow{3}{*}{$\begin{array}{l}\text { Weakness and difficulty in grasping severity } \\
\text { by BCTQ }\end{array}$} & \multicolumn{4}{|c|}{ Control group $(n=15)$} & \multicolumn{4}{|c|}{ Study group $(n=15)$} \\
\hline & \multicolumn{2}{|c|}{ pre-treatment } & \multicolumn{2}{|c|}{ post-treatment } & \multicolumn{2}{|c|}{ pre-treatment } & \multicolumn{2}{|c|}{ post-treatment } \\
\hline & No. & $\%$ & No. & $\%$ & No. & $\%$ & No. & $\%$ \\
\hline No weakness or difficulty in grasping & 0 & & 0 & & 0 & & 15 & 100 \\
\hline Little weakness and difficulty in grasping & 1 & 6.67 & 6 & 40 & 0 & & 0 & \\
\hline Moderate weakness and difficulty in grasping & 8 & 53.33 & 8 & 53.33 & 7 & 46.67 & 0 & \\
\hline Intense weakness and difficulty in grasping & 4 & 26.67 & 1 & 6.67 & 6 & 40 & 0 & \\
\hline Severe weakness and difficulty in grasping & 2 & 13.33 & 0 & & 2 & 13.33 & 0 & \\
\hline Range & \multicolumn{2}{|c|}{$1-4$} & \multicolumn{2}{|c|}{$1-3$} & \multicolumn{2}{|c|}{$1-4$} & \multicolumn{2}{|c|}{0} \\
\hline Median & \multicolumn{2}{|c|}{2} & \multicolumn{2}{|c|}{2} & \multicolumn{2}{|c|}{2} & \multicolumn{2}{|c|}{0} \\
\hline$z$ value & \multicolumn{4}{|c|}{-2.521} & \multicolumn{4}{|c|}{-3.446} \\
\hline$p$ value & \multicolumn{4}{|c|}{$>0.05$} & \multicolumn{4}{|c|}{$<0.001$} \\
\hline
\end{tabular}

CTS - carpal tunnel syndrome, BCTQ - Boston Carpal Tunnel Questionnaire

$(p<0.001)$ within the study group. The post-treatment comparison of both groups revealed a statistically highly significant decrease in weakness and difficulty in grasping severity $(p<0.001)$ in favour of the study group (Table 5$)$.

\section{Discussion}

Women commonly experience CTS during pregnancy as a result of water retention, and although symptoms generally resolve after giving birth, mild symptoms may still be experienced after 3 years [2]. Tingling, pins and needles, numbness, pain, or burning sensations along the median nerve course are the general characteristics of CTS [9]. Pain may often worsen at night, causing waking [10]. Longstanding CTS causes irreversible nerve damage, atrophy of the thenar eminence and some hand muscles, and weakness of the palmar abductor [11]. The current study was conducted to determine the effect of myofascial release on electrophysiological and clinical measures in pregnant wom-
Regarding the control group, the results showed a statistically significant decrease in numbness and tingling sensation, whereas there was a statistically non-significant reduction in median nerve DML, pain intensity, weakness and difficulty in grasping severity between pre- and post-treatment.

The significant decrease in numbness could be attributed to the effect of wearing a night wrist splint, and that is supported by Werner and Andary [12], who reported that short-term night splinting might decrease wrist, hand, and/ or finger discomfort among active workers with CTS symptoms. In addition, Manente et al. [13] suggested that wearing a night wrist splint for 4 weeks was more effective than no treatment in relieving the symptoms of CTS. Akalin et al. [9], in turn, found a non-significant difference in the improvement of CTS symptoms between patients treated only with splints and those treated with splints along with nerve and tendon gliding exercises.

As for the best timing of splint wearing for improvement of CTS symptoms, Manente et al. [13] reported great effects of only night splinting with stretching the middle and ring 
fingers. On the other hand, Piazzini et al. [14] proved that splinting was most efficient when applied for the whole day. However, Halac et al. [15] recommended night splint wearing for patients who had only night symptoms, and full-time splint wearing for those with persistent symptoms. Finally, Al-Muhanna and Shamsi [16] observed a non-significant difference between night splinting and day splinting in relieving CTS pain in women. They concluded that CTS patients should apply splinting at their most convenient time.

Concerning the nerve conduction study for CTS, there was a non-significant decrease in median nerve DML, mostly the same for all sensations except numbness. This can be explained by observations by Kruger et al. [17], who showed motor latency improvement in the symptom relief group, while there was motor latency deterioration in the non-relief group; the motor latency improvement was possibly associated with the patient's perception of relief. In contrast, Weng et al. [18] reported a significant decrease in median nerve DML and both SSS and FSS. They concluded that neutral wrist splinting at night was an effective method for CTS patients treatment.

With reference to the study group, the results showed a statistically highly significant decrease in median nerve DML, pain intensity, numbness and tingling sensation severity, and weakness and difficulty in grasping severity between pre- and post-treatment.

These results remain in line with a study by Sucher [19], who found a clinical improvement of CTS in patients following myofascial release. The clinical improvement was confirmed with magnetic resonance imaging, which demonstrated that the anteroposterior and transverse dimensions of the carpal tunnel had significantly increased post-treatment; in turn, nerve conduction studies revealed a significant decrease in distal latencies.

Also, the results of this study were consistent with those obtained by Bordoni and Zanier [20], who reported that myofascial release was a widely employed direct manual medicine treatment which applied specifically directed mechanical forces for manipulating and decreasing myofascial restrictions in different somatic dysfunctions. The combination of myofascial release with other conventional treatment is effective to produce immediate pain relief.

In addition, the present results agreed with the observations by Paolini [21], who attributed the significant reduction in pain intensity to personal attention and human touch that commonly decreases the pain perception. This links to the parasympathetic response of the autonomic nervous system. The stimulation of the parasympathetic response reduces stress hormones release, anxiety, depression, and pain. The serotonin release blocks the noxious stimuli transmission to the brain. The release of other inhibitory neurotransmitters, such as endorphins, may be stimulated by the pressure that is generated by the treatment.

Moreover, a case report and literature review by Robb and Sajko [22] supports the results of the present study as it concluded that myofascial release techniques could take a part in treating peripheral nerve entrapments. In that case report, an elite baseball pitcher experienced pain in the elbow and numbness along the dorsum side of his hand, mainly the thumb and index fingers. The clinical impression for that case was nerve compression from the decelerating muscles used during the throwing motion. When these muscles undergo eccentric loading, they can compress or apply extreme tensile loading along the radial nerve course. Treatment for this injury comprised rest and myofascial release to the involved muscles.
Regarding the comparison between both groups posttreatment, the results revealed a statistically significant decrease in the median nerve DML, pain intensity, numbness and tingling sensation severity, and weakness and difficulty in grasping severity in favour of the study group.

The review of literature did not detect any study addressing the combination of myofascial release and wrist splint in the treatment of CTS among pregnant women. Therefore, the present work is considered the first study on this point. Accordingly, the results cannot be compared or discussed with other research outcomes but showed a significant effect of myofascial release on treating CTS during pregnancy.

\section{Limitations}

Although the current study reveals objective data with statistically significant differences, there are some limitations. The main one is the small sample size so larger studies are necessary to confirm the findings. Also, the study duration was short. Therefore, longitudinal studies are needed to evaluate a long-term effect of myofascial release on electrophysiological and clinical measures in pregnant women with CTS.

\section{Conclusions}

Myofascial release is considered a safe, non-invasive, and simple method for decreasing pain intensity and numbness sensation severity and for improving hand function in pregnant women with CTS.

\section{Acknowledgments}

We would like to thank all individuals who contributed to the completion of this work, especially the study participants.

\section{Disclosure statement}

No author has any financial interest or received any financial benefit from this research.

\section{Conflict of interest}

The authors state no conflict of interest.

\section{References}

1. Khosrawi S, Maghrouri R. The prevalence and severity of carpal tunnel syndrome during pregnancy. Adv Biomed Res. 2012;1:43; doi: 10.4103/2277-9175.100143.

2. Mondelli M, Rossi S, Monti E, Aprile I, Caliandro P, Pazzaglia C, et al. Long term follow-up of carpal tunnel syndrome during pregnancy: a cohort study and review of the literature. Electromyogr Clin Neurophysiol. 2007;47(6): 259-271.

3. Finsen V, Zeitlmann H. Carpal tunnel syndrome during pregnancy. Scand J Plast Reconstr Surg Hand Surg. 2006; 40(1):41-45; doi: 10.1080/02844310500410351.

4. Viera AJ. Management of carpal tunnel syndrome. Am Fam Physician. 2003;68(2):265-272.

5. Sucher BM. Palpatory diagnosis and manipulative management of carpal tunnel syndrome. J Am Osteopath Assoc. 1994;94(8):647-663; doi: 10.7556/jaoa.1994.94. 8.647.

6. Chila AG. Foundations for osteopathic medicine, $3^{\text {rd }}$ ed. Philadelphia: Lippincott Williams \& Wilkins; 2010.

7. Donahue JE, Raynor EM, Rutkove SB. Forearm velocity in carpal tunnel syndrome: when is slow too slow? Arch Phys Med Rehabil. 1998;79(2):181-183; doi: 10.1016/ S0003-9993(98)90297-0. 
8. Sambandam SN, Priyanka P, Gul A, llango B. Critical analysis of outcome measures used in the assessment of carpal tunnel syndrome. Int Orthop. 2008;32(4):497504; doi: 10.1007/s00264-007-0344-7.

9. Akalin E, El O, Peker O, Senocak O, Tamci S, Gülbahar S, et al. Treatment of carpal tunnel syndrome with nerve and tendon gliding exercises. Am J Phys Med Rehabil. 2002;81(2):108-113; doi: 10.1097/00002060-20020200000006.

10. Verdugo RJ, Salinas RA, Castillo JL, Cea JG. Surgical versus non-surgical treatment for carpal tunnel syndrome. Cochrane Database Syst Rev. 2008;4:CD001552; doi: 10.1002/14651858.CD001552.pub2.

11. Uemura T, Hidaka N, Nakamura H. Clinical outcome of carpal tunnel release with and without opposition transfer. J Hand Surg Eur Vol. 2010;35(8):632-636; doi: $10.1177 / 1753193410369988$.

12. Werner RA, Andary M. Carpal tunnel syndrome: pathophysiology and clinical neurophysiology. Clin Neurophysiol. 2002;113(9):1373-1381; doi: 10.1016/S13882457(02)00169-4.

13. Manente G, Torrieri F, Di Blasio F, Staniscia T, Romano F, Uncini A. An innovative hand brace for carpal tunnel syndrome: a randomized controlled trial. Muscle Nerve. 2001;24(8):1020-1025; doi: 10.1002/mus.1105.

14. Piazzini DB, Aprile I, Ferrara PE, Bertolini C, Tonali P, Maggi $L$, et al. A systematic review of conservative treatment of carpal tunnel syndrome. Clin Rehabil. 2007; 21(4):299-314; doi: 10.1177/0269215507077294.

15. Halac G, Demir S, Yucel H, Niftaliyev E, Kocaman G, Duruyen $\mathrm{H}$, et al. Splinting is effective for night-only symptomatic carpal tunnel syndrome patients. J Phys Ther Sci. 2015;27(4):993-996; doi: 10.1589/jpts.27.993.

16. Al-Muhanna NA, Shamsi S. Different splinting time for carpal tunnel syndrome in women: comparative study. IOSR J Nurs Health Sci. 2015;4(2):3-11; doi: 10.9790/ 1959-04210311.

17. Kruger VL, Kraft GH, Deitz JC, Ameis A, Polissar L. Carpal tunnel syndrome: objective measures and splint use. Arch Phys Med Rehabil. 1991;72(7):517-520.

18. Weng C, Dong H, Chu H, Lu Z. Clinical and electrophysiological evaluation of neutral wrist nocturnal splinting in patients with carpal tunnel syndrome. J Phys Ther Sci. 2016;28(8):2274-2278; doi: 10.1589/jpts.28.2274.

19. Sucher BM. Myofascial manipulative release of carpal tunnel syndrome: documentation with magnetic resonance imaging. J Am Osteopath Assoc. 1993;93(12): 1273-1278; doi: 10.7556/jaoa.1993.93.12.1273.

20. Bordoni B, Zanier E. Understanding fibroblasts in order to comprehend the osteopathic treatment of the fascia. Evid Based Complement Alternat Med. 2015;2015: 860934; doi: 10.1155/2015/860934.

21. Paolini J. Review of myofascial release as an effective massage therapy technique. Athl Ther Today. 2009;14(5): 30-34; doi: 10.1123/att.14.5.30.

22. Robb A, Sajko S. Conservative management of posterior interosseous neuropathy in an elite baseball pitcher's return to play: a case report and review of the literature. J Can Chiropr Assoc. 2009;53(4):300-310. 